\title{
MEAN-FIELD DESCRIPTION OF STRUCTURAL PHASE TRANSITIONS IN A HYPERCUBIC LATTICE MODEL
}

\author{
S. E. Mkam Tchouobiap* and H. Mashiyama \\ Department of Physics, Graduate School of Science and Engineering, \\ Yamaguchi University, Yamaguchi 753-8512, Japan
}

(Received May 15, 2006, finally revised July 29, 2006)

We examine the mean-field theory investigation of a hypercubic lattice model that exhibits structural phase transitions. In this model, with anharmonicity as main characteristic and displacement-displacement stiffness constant, the occurrence of phase transitions mechanism is analyzed and the effects of the anharmonicity on the order of the transition are studied. Discussing the features of the Hamiltonian, thermodynamic properties calculated show the occurrence of the structural phase transitions, which are either (i) a second-order transition (characteristic of short-range attractive part of the double- Morse one-site potential) or (ii) a first-order transition with sharp melting transition and bifurcation entropy, depending on the strength of the anharmonicity.

Keywords: Mean-field theory, structural phase transition, D-dimensional hypercubic lattice model, anharmonicity. 


\section{S. E. Mkam Tchouobiap and H. Mashiyama}

\section{INTRODUCTION}

Since a pioneering work by Landau on structural phase transitions, the diversity of both the categories of phase transitions and mechanism for causing them continue to motivate further research; particularly the first order structural phase transitions where the observed microscopic mechanisms responsible for the macroscopic features continue to warrant and receive further attention [1-4, 6]. More recent research have focused and emphasized the role of the large amplitude fluctuations that precede the transition and the intrinsically nonlinear mechanisms which are needed to describe such fluctuations $[3,6]$. All this led to a search of others models possessing mechanisms which produce structural transitions and particularly discontinuous ones. Then, many intuitive models and theories have been introduced and studied. In order to concretely study such transitions, statistical-mechanical models must incorporate the change in effective restoring forces between particles at the transition, as this coupling controls the vibrational spectrum $[4,5]$. Usually, the interaction energy between lattice sites, taken to be harmonic and represented by a strictly convex function of the form $[2-3,6]$

$$
V_{2}\left(q, q^{\prime}\right)=\frac{1}{2} K\left(q-q^{\prime}\right)^{2}
$$

appears to be not sufficient, as the coupling does not change at the transition.

In order to understand the mechanism of structural phase transitions, the conditions that a system must fulfill to exhibit such a transition, and the effects of interparticle anharmonicty on the order of the phase transition, we propose a D-dimensional hypercubic lattice model, with a symmetric double Morse-type on-site potential and anharmonic nearest-neighbors interaction potential, that exhibits structural phase transitions and particularly first-order one. The essentials features of our lattice-dynamical model are that (i) each particle moves in an symmetric double-well potential and (ii) the interparticle interactions are anharmonic. Consequently, nonlinear forces acting on each particle arise both from the on-site potential

\footnotetext{
*Corresponding author, e-mail: h501hr@yamaguchi-u.ac.jp
} 
and the interactions with neighboring particles. The intersite coupling which models the cooperative effects is introduced at the microscopic level, and reflects the change in the electronic distribution on the particles. This idea derives from an argument due to Zener showing the importance of the vibrational contribution to the entropy to the transitions [4]. The mean-field theory (MFT) is developed for this model which incorporates the change in effective restoring force between particles at the transition. This anharmonicity in the interparticle introduces a competing factor and the system shows a sharp melting transition. Although we cannot claim that this is a true first-order transition, it exhibits close similarities. Thus, for sufficiently strong anharmonicity, when the temperature is increased to a critical one, the structure with the higher internal energy achieves the lower free energy and discontinuous change to that structure occurs. Although such a model Hamiltonian was recently proposed by us [7], our aim here is to display the interesting dynamics obtained in this model, whose anharmonicity is the main characteristic and discuss the origin of this particular behavior.

\section{MODEL AND MEAN-FIELD THEORY}

The lattice model Hamiltonian exhibiting the phase transition we consider is giving by [7]:

$$
H=\sum_{n}\left[\frac{1}{2} \frac{p_{n}^{2}}{m}+V_{1}\left(q_{n}\right)\right]+\frac{1}{2} \sum_{n, \delta} V_{2}\left(q_{n}, q_{n+\delta}\right),
$$

where $n$ denotes the sites of a D-dimensional hypercubic lattice, $\delta$ denotes the set of nearest-neighbor lattice vectors and $q_{n}$ is a scalar displacement variable at the $n$th lattice site. $p_{n}$ is the momentum of the $n$th lattice site of mass $m$ which moves in the double-well anharmonic potential of the double Morse-type. The expression of this on-site potential energy $V_{1}\left(q_{n}\right)$ is

$$
V_{1}\left(q_{n}\right)=V_{0}\left[A \cosh \left(\alpha q_{n}\right)-1\right]^{2}-\frac{1}{2} V_{0}\left(A^{2}+2\right)
$$




\section{S. E. Mkam Tchouobiap and H. Mashiyama}

with

$$
A=2 \exp \left[-\alpha\left(\frac{l}{2}-r_{0}\right)\right]
$$

where $V_{0}, A$ and $\alpha$ are the positive parameters of the potential, and $l$ is the equilibrium lattice spacing. It should be noted that $0<A<1$ in order to ensure a double-well form of $V_{1}\left(q_{n}\right)$, which is formed by superposing the back-to-back two Morse potentials $[8,9]$. $\alpha$ and $r_{0}$ are the phenomenological parameters of the initial Morse [8, 10]. Also $\alpha^{-1}$ fixes the length over which the nonlinearity of the potential shows up while $V_{0}$, which is the dissociation energy, sets the energy scale. The value $\Delta V=V_{0}(1-A)^{2}$ corresponds to the potential barrier height between the two minima of the double-well whose positions $\pm q_{0}$ are given by the equation $\cosh \left(\alpha q_{0}\right)=1 / A$.

For the interaction potential term between neighboring lattice sites, we have used the following anharmonic function [11]

$$
V_{2}\left(q, q^{\prime}\right)=\frac{1}{2} K\left\{1+\rho \exp \left[-a\left(q+q^{\prime}\right)\right]\right\}\left(q-q^{\prime}\right)^{2}
$$

where $K$ is the stiffness constant of the intersite interaction. This intersite coupling, replacing the simple usual harmonic one of past several approaches, $[1-4,6,8]$ is one of essential features of the model, and it is responsible for its interesting properties. $a$ and $\rho$ are the anharmonicity parameters. It is also interesting to know that this interaction potential energy could be harmonic if $a=0$ or $\rho=0$ with interparticle harmonic force constant $K(1+\rho)$ and $K$, respectively. The essential idea is to stabilize the higher-energy structure at higher temperatures by having higher vibrational entropy. The function $V_{2}\left(q, q^{\prime}\right)$ achieves this effect by decreasing the effective force coupling "constant” from $K(1+\rho)$ to $K$ when either one of the two interacting sites is stretched. The motivation for choosing this model energy potential function is given in detail in [7]. Our major concern is to see how the system properties change with increasing anharmonicity strength. For convenience, we introduce the following units: $l$ for displacement, $m$ for mass, $\tau=t \sqrt{K / M}$ for time, and $K l^{2}$ for energy and where the following dimensionless parameters $\bar{H}=H / K l^{2}, \varepsilon_{0}=V_{0} / K l^{2}, u_{n}=q_{n} / l$, 
$P_{n}=p_{n} / \sqrt{m K l^{2}}, T=k_{B} T / K l^{2}, \beta=\alpha l$, and $\lambda=a l$ are introduced.

Mean-field theory assumes that the particles move independently, so that the phase-space probability distribution function factors into single-particle functions [4].

Following the ansatz originally used by Thomas [12], we get the following expressions of the internal energy $U$ and entropy $S$ per particle

$$
\begin{aligned}
& U=\frac{\langle\bar{H}\rangle}{N}= \frac{1}{2} T+\varepsilon_{0}\left[\frac{A^{2}}{2} \exp \left(2 \beta^{2} \sigma\right) \cosh (2 \beta \eta)-2 A \exp \left(\frac{\beta^{2} \sigma}{2}\right) \cosh (\beta \eta)\right] \\
&+D\left(1+\rho \exp \left(-2 \lambda \eta+\lambda^{2} \sigma\right)\right), \\
& S=\frac{1}{N}\left\langle\ln F_{N}\right\rangle=1+\frac{1}{2} \ln (2 \pi T)+\frac{1}{2} \ln (2 \pi \sigma),
\end{aligned}
$$

where we have used the fact that the coordination number of the D-dimensional hypercubic lattice is $2 \mathrm{D}$ (2D nearest-neighbor lattice vectors) $[4,13]$ and the translational invariance. Here, $\eta$ and $\sigma$, which are respectively the average displacement $<u_{\mathrm{n}}>$ (related to order parameter and its variance $<\left(u_{\mathrm{n}}-<u_{\mathrm{n}}>\right)^{2}>$ (mean-square fluctuation), represent variational parameters.

By using the thermodynamic relation and equations (6) and (7), the free energy per particle $f=U-T S$ is minimized with respect $\eta$ and $\sigma$, and we obtain

$$
\begin{gathered}
\beta \varepsilon_{0}\left[A^{2} \exp \left(2 \beta^{2} \sigma\right) \sinh (2 \beta \eta)-2 A \exp \left(\frac{\beta^{2} \sigma}{2}\right) \sinh (\beta \eta)\right]-2 D \lambda \sigma \rho \exp \left(-2 \lambda \eta+\lambda^{2} \sigma\right)=0 \\
\beta^{2} \varepsilon_{0}\left[A^{2} \exp \left(2 \beta^{2} \sigma\right) \cosh (2 \beta \eta)-A \exp \left(\frac{\beta^{2} \sigma}{2}\right) \cosh (\beta \eta)\right]-\frac{T}{2 \sigma} \\
+D\left[1+\rho\left(1+\lambda^{2} \sigma\right) \exp \left(-2 \lambda \eta+\lambda^{2} \sigma\right)\right]=0
\end{gathered}
$$

These equations have to be solved self-consistently and analyzed owing the equilibrium values of $\eta$ and $\sigma$. The solutions along with the free energy constitute the MFT description of the statistical mechanics of the system.

\section{RESULTS AND DISCUSSIONS}




\section{S. E. Mkam Tchouobiap and H. Mashiyama}

Now, we will present in this section the solutions of these MFT equations including interparticle anharmonicity interactions and analyze the state of the system, depending upon parameters.

A reasonable result that there are no fluctuations about the equilibrium position at zero temperature is showed by Eq. (9) that, in the limit $T \rightarrow 0$, the ratio $T / \sigma$ remains finite and so it must be that $\sigma \rightarrow 0$ also. Then Eq.(8) is reduced to $d V_{1}(\eta) / d \eta=0$ which states that at $T=0$, the average position is one of the positions of mechanical equilibrium of on-site potential.

At finite temperature, these equations do not admit trivial solutions and are difficult to solve analytically due to their implicit characters, so we will try to solve them numerically. For simplicity, the values of the model parameters used for the graphs are: $A=0.5, \alpha=8.2$, $D=2, \varepsilon_{0}=0.2$ and $\rho=1$, where the set of double Morse potential result from the analysis of geometric and ab initio lattice characteristics of the $\mathrm{O}-\mathrm{H} \cdots \mathrm{O}$ bonded ferroelectrics [13-15].

Figure 1 shows the numerical solution of the absolute temperature dependence of the order parameter $\eta$ for 2 different values of $\lambda$. In spite the fact that the solution with lower free energy evolves out of the two minima of the on-site potential as $\mathrm{T}$ increases from zero and that the two branches of the curves are important, the physical solution for $\eta$ is the top branch in Fig.1 [4, 7]. Then it appears that the top branch of $\eta$ vs $T$ becomes steeper as $\lambda$ decreases. However, the bottom branch is itself double valued and intercepts the $T=0$ axis at both $\eta=0$ and $\eta=-\cosh ^{-1}(1 / A) / \beta$. One can visualize from Fig. 1 that there exits a critical value $\lambda_{c}$ at which the top branch of $\eta$ develops an infinite slope at a certain $T$, showing the appearance of the critical point $[14,16,17]$. This critical value of $\lambda_{c}$ depends on the values of other parameters. As $\lambda$ continues to decrease, for smaller $\lambda$ values the top branch of the $\eta$ vs $T$ graph folds back and becomes multivalued over a certain temperature interval, so that a discontinuity of the order parameter $\eta$ develops $[16,18]$. Fig. 1(b) shows the solution for $\lambda=0.2$, where the multivalued nature is evident.

To compare in more details, predictions of the theory, the corresponding free energy function and entropy are shown in figures 2 and 3 . The branch of $f$ corresponding to the top 
branch of $\eta$ vs $T$ graph (Fig. 1) always has lower values than the branch of $f$ corresponding to the bottom branch $\eta$ vs $T$, and so it is the equilibrium solution (equilibrium state). The corresponding free energy graphs develop a narrow loop which amplitude increases as $\lambda$ decreases (large for the first-order transitions, Fig. 2(b)). On the temperature interval where the top branch of $\eta$ vs $T$ graph is multivalued, the loop is similar to the loop of free energy function in the van der Waals theory of nonideal gas.[19] Since the thermal equilibrium state is the one of minimum $f$, the transition is at the temperature $T_{c}$ where the free energy graph intercepts itself $[4,5,18]$. At this temperature the system undergoes a change in $\eta$ which must be continuous (second-order) or discontinuous (first-order). The discontinuity is characterized by a jump between two points of the same solution branch of the MFT equations. Then parts of the loop may be metastable achievable by superheating or supercooling and are solutions of these MFT equations. Also the critical value $\lambda_{c}$, where the transition first develops, is the value for which the first and second derivatives of temperature with respect to average displacement $\eta$ vanish, and have common solution for $\eta>0$.

These equations along with Eq. (9) determine $\lambda_{\mathrm{c}}$ and the values of $T_{\mathrm{c}}$ and $\eta$ where the transition initially occurs. Figure 3 shows the $T$-dependence of entropy $s=-\partial f / \partial T$; so it increases discontinuously at the transition for the first-order structural phase transition (Fig. 3(b)) and increases continuously at low temperature (Fig. 3(a)). Then it appears that the anharmonicity of the interparticle interactions makes the lattice vibrational frequencies dependent on the structure parameters [20] and this dependence provides the mechanism which creates a bifurcation on the high energy structure relative to the low energy-one and also on entropy. 


\section{S. E. Mkam Tchouobiap and H. Mashiyama}

\section{References}

[1] L. D. Landau and E. M. Lifshitz, Course of Theoretical Physics, Statistical Physics (Pergamon Press, Oxford, 1986) part I.

[2] A. D. Bruce and R. A. Cowley, Structural Phase Transitions (Taylor and Francis, London, 1981).

[3] E. K. H. Salje, B. Wruck and H. Thomas, Z. Phys. B 82, 399 (1991).

[4] W. C. Kerr and M. J. Rave, Phys. Rev. B 48, 16234 (1993).

[5] W. C. Kerr, A. M. Hawthorne, R. J. Gooding, A. R. Bishop and J. A. Krumhansl, Phys. Rev. B 45, 7036 (1992).

[6] E. Allroth and H. Muller-Krumbhaar, Phys. Rev. A 27, 1575 (1983).

[7] S. E. Mkam Tchouobiap, C. M. Ngabireng and T. C. Kofane, Phys. Scr. 73, 223 (2006).

[8] H. Konwent, Phys. Status Solidi b 138, K7 (1986).

[9] O. Yanovitskii, G. Vlastou-Tsinganos, and N. Flytzanis, Phys. Rev. B 48, 12645 (1993).

[10] S. Tanaka, Phys. Rev. B 42, 10488 (1990).

[11] T. Dauxois and M. Peyrard, Phys. Rev. E 51, 4027 (1995).

[12] H. Thomas, Structural Phase transitions and Soft Modes, edited by E. J. Samuelson, E. Anderson and J. Feder (Universitetsforlaget, Oslo, 1971), p.15.

[13] O.Yanovitskii, G. Vlastou-Tsinganos and N. Flytzanis, Phys. Rev. B 48, 12645 (1993).

[14] R. Blinc, B. Zeks, J. F. Sampio, A. S. T. Pires and F. C. Sa Barreto, Phys. Rev. B 20, 1991 (1979).

[15] Q. Zang, N. Kioussis, S. G. Demos, and H. B. Radousky, J. Phys.: Condens. Matter. 14, L89 (2002).

[16] Y. Moreno, G. B. Gomez and A. F. Pacheco, Phys. Rev. Lett. 85, 2865 (2000).

[17] Yu. L. Klimontovich, Int. J. Bifurcation Chaos. 8, 665 (1998).

[18] E. A. Shadchin and A. I. Barabash, J. Mol. Struct. 524, 283 (2000).

[19] See, e.g., C. Kittel and H. Kroemer, Thermal Physics (Freeman, San Francisco, 1980) p. 293.

[20] J. R. Morris and R. J. Gooding, Phys. Rev. Lett. 65, 1769(1990). 
Figure 1. Temperature dependence of the order parameter (average displacement) for models parameters and for two different values of anharmonicity parameter $\lambda$ : (a) $\lambda=8.0$ and (b) $\lambda=0.2$. Broken lines indicate the second-order transition for the case of $\rho=0$.
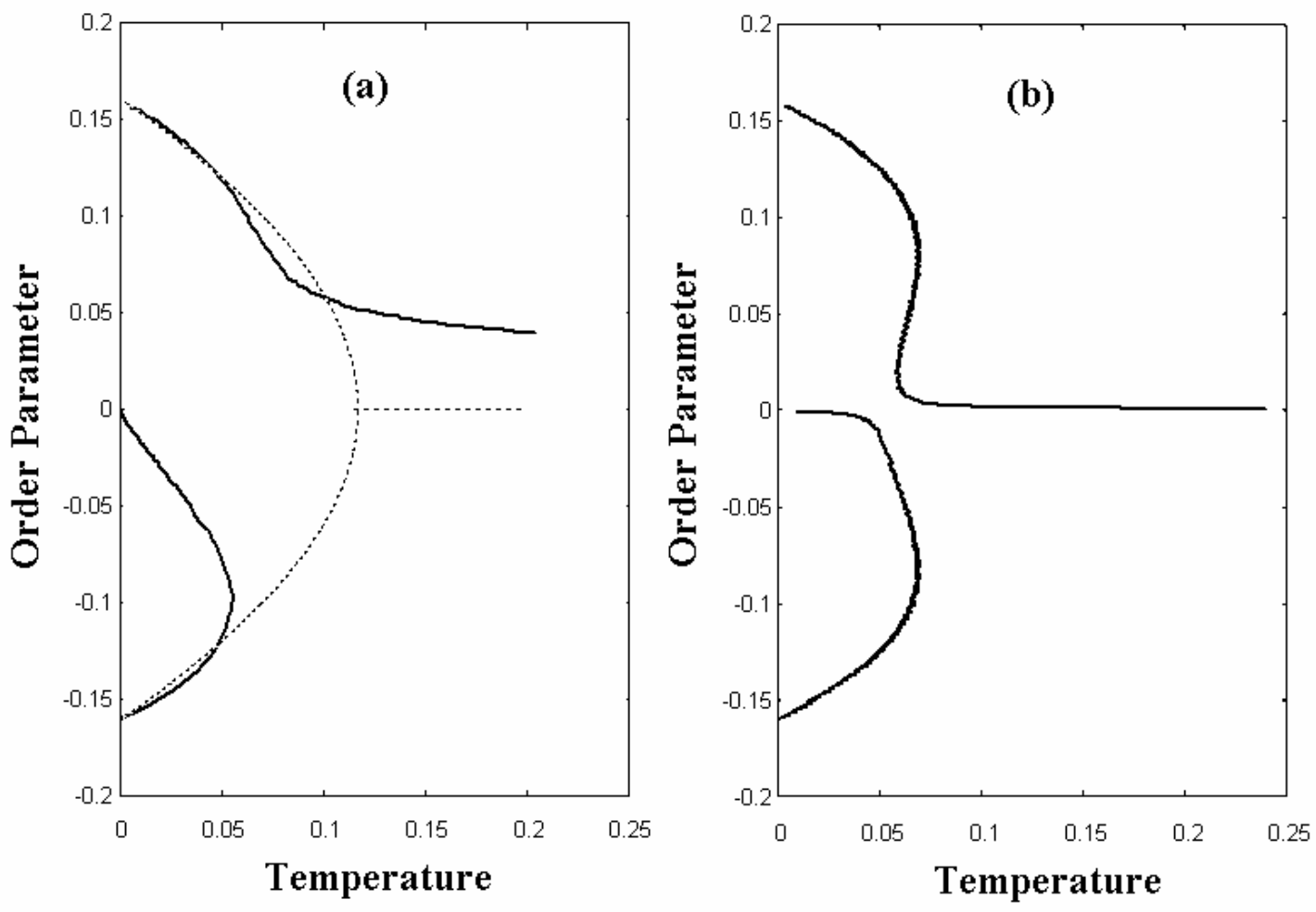


\section{S. E. Mkam Tchouobiap and H. Mashiyama}

Figure 2. Free energy versus temperature corresponding to average displacement graphs in figure 1 for (a) $\lambda=8.0$ and (b) $\lambda=0.2$ respectively. The solid curve corresponds to the top branch of Fig. 1 and contains the equilibrium solution. The dashed curve corresponds to the bottom branch of Fig. 1, and is a very narrow loop in the first-order transition case ((b) $\lambda=0.2)$.
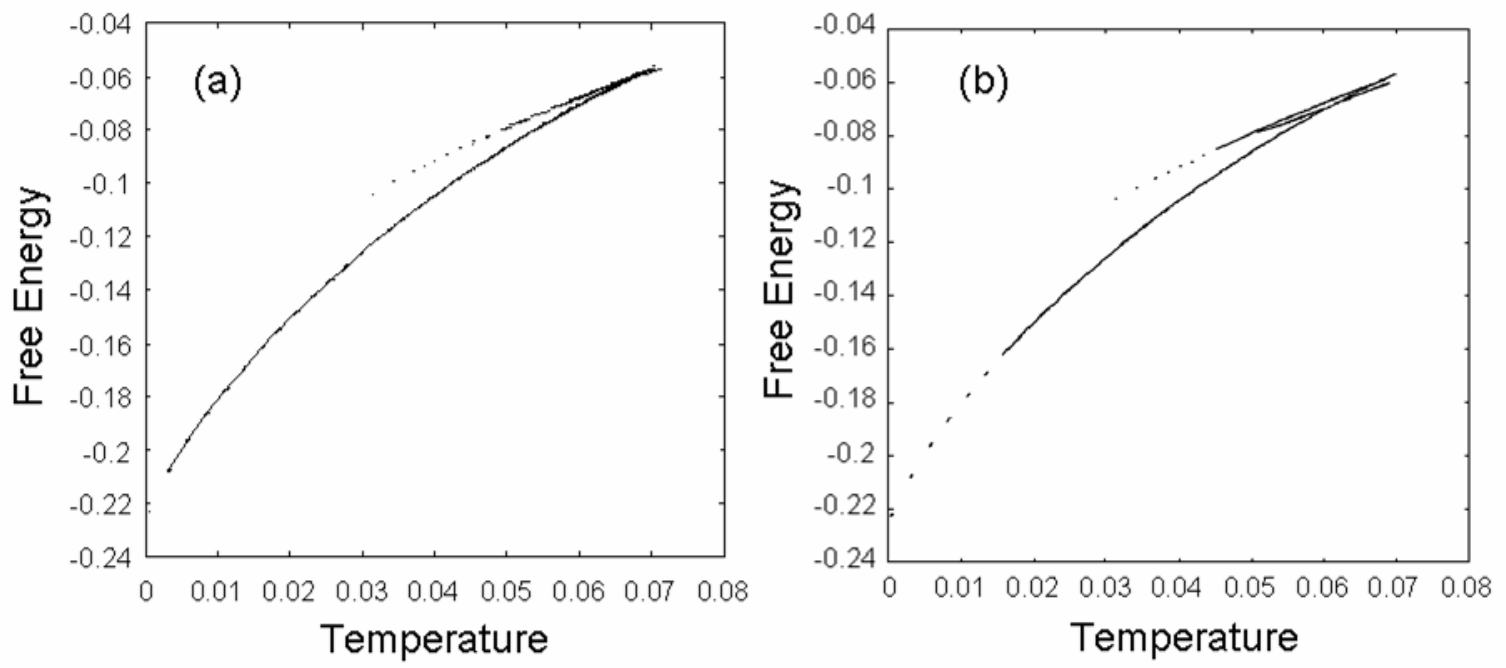

Figure 3. Entropy per particle versus temperature for two different anharmonicity parameter $\lambda$ values: (a) $\lambda=8.0$ and (b) $\lambda=0.2$.
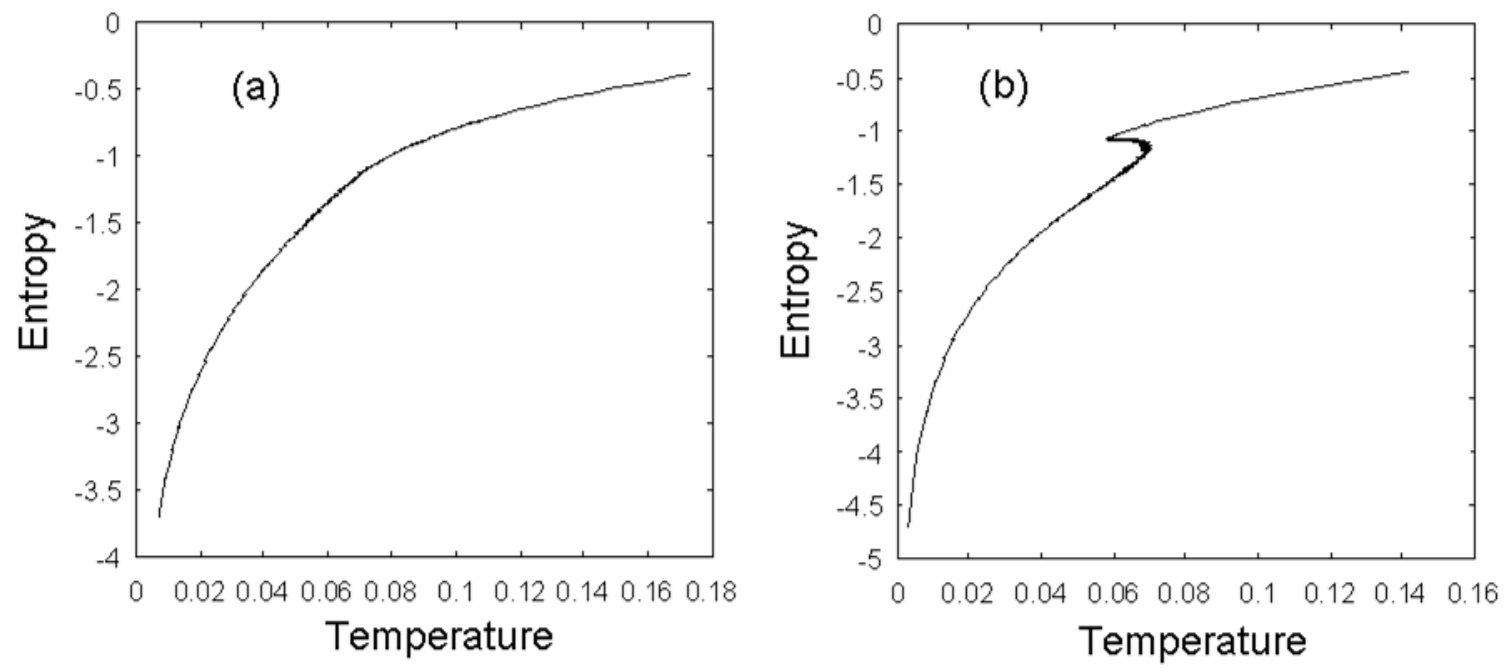